\title{
La eVOLUCIÓN DE LA CIENCIA POLÍTICA EN ChILE: UN ANÁLISIS EXPLORATORIO (1980-2000)*
}

\author{
Alfredo Rehren \\ Instituto de Ciencia Política, Pontificia Universidad Católica de Chile, Chile \\ Marco Fernández \\ Escuela de Ciencia Politica, Universidad Arcis, Chille
}

\begin{abstract}
Resumen
Este artículo intenta llenar un vacío en el conocimiento de la evolución de la disciplina desde su temprano inicio en la década de los 60. Para hacerlo, analiza las publicaciones de revistas ya establecidas, la producción de tesis de postgrado y el volumen y tipo de proyectos de investigación emprendidos con recursos públicos desde los 80 . Examinando los campos tradicionales de especialización en la disciplina - teoría política, relaciones internacionales, instituciones políticas, comportamiento político, políticas públicas e historia política- los autores concluyen que, en una disciplina crecientemente institucionalizada, existe un desequilibrio en su desarrollo y productividad entre los diferentes campos de especialidad como también al interior de ellos, donde existe una relación desigual entre publicaciones, tesis de postgrado y proyectos de investigación.
\end{abstract}

Abstract

This article attempts to fill in a gap in the knowledge of the evolution of the discipline since its early inception in the 1960s. In so doing, it analyzes publications in established journals, ouput in graduate thesis and volume and type of research undertaken with public funds since the 1980s. Looking at the traditional fields of specialization in the discipline - political theory, international relations, political institutions, political behavior, public policy and political history- the authors conclude that in an increasing institutionalizing discipline, there is an unbalanced development and output among fields and within fields of specialization.

\section{PALABRAS CLAVE - Ciencia Política - Chile • Publicaciones - Investigaciones - Productividad Disciplinaria}

\section{INTRODUCCIÓN}

Considerados como grupo técnico-profesional, los cientistas políticos carecen de integración y de homogeneidad en los enfoques y estrategias de trabajo... [y] no han llegado aún a ser un grupo profesional reconocido y valorado en las sociedades de América Latina (Kaplan, 1970: 50 y 53). 
Los estudios sobre la evolución de la ciencia política en países donde ésta se encuentra más desarrollada, incorporan una serie de dimensiones entre las cuales se destacan la inserción de la disciplina en las instituciones educacionales nacionales; el nivel académico de profesores e investigadores; la existencia de centros de investigación; el apoyo y recursos disponibles para la investigación; la existencia de asociaciones y reuniones profesionales; la calidad de las publicaciones y finalmente las oportunidades que los cientistas políticos tienen de hacer una carrera profesional y/ 0 académica ${ }^{1}$.

Lo anterior debe ser puesto, necesariamente, en el contexto socio-cultural nacional, la naturaleza de experiencias políticas concretas, el nivel de desarrollo socio-económico logrado y la naturaleza del clima intelectual vigente. Asimismo, los conflictos entre escuelas y paradigmas que han competido por explicar la naturaleza del fenómeno político chileno, la relación de la Ciencia Política con otras ciencias sociales, la inserción de la disciplina en el contexto internacional y, por sobre todo, la naturaleza del conocimiento generado por la disciplina son igualmente dimensiones a considerar.

Para evaluar el aporte de la Ciencia Política al desarrollo de la sociedad es necesario determinar, además, el posicionamiento, grado de influencia y prestigio que los cientistas políticos tienen en el ámbito universitario nacional y público. Pero se puede argumentar que, siguiendo la experiencia de otros países, la politología chilena se consolidará disciplinariamente en la medida que se produzcan generaciones de investigadores que se identifiquen como cientistas políticos y que ubicados en centros de producción académica gocen de estabilidad para desarrollar sistemática y rutinariamente sus trabajos, promuevan sus ideas e investigaciones y publiquen sus resultados.

Este trabajo tiene por objeto analizar algunas de estas dimensiones y en particular estructurar el análisis alrededor de las siguientes preguntas: ¿Cuándo y dónde se abre el campo de estudio en la Ciencia Política en Chile? ¿Cómo evoluciona institucionalmente la disciplina? ¿Cuáles son las principales publicaciones profesionales a través de las cuales se difunde el conocimiento de lo político? ¿Cuál es el rol de la investigación y qué se investiga? ¿Qué temas investigan las futuras generaciones de cientistas políticos?

Transcurridos ya 50 años desde que se impartieron los primeros cursos en Ciencia Política y 40 desde que la revolución conductista impactó en la naciente disciplina, este trabajo aporta datos empíricos y discute en forma exploratoria la evolución de las principales tendencias de la Ciencia Política chilena. ¿Hemos evolucionado en Chile más allá del sombrío cuadro pintado por Kaplan en nuestra cita introductoria?

\section{LA INSTITUCIONALIZACIÓN DE LA CIENCIA POLÍTICA EN CHILE}

La Ciencia Política aparece como foco de atención disciplinario y profesional en la Escuela de Ciencias Políticas y Administrativas de la Universidad de Chile donde se impartieron los primeros cursos en Ciencia Política a mediados de los años 50. Formados en la tradición francesa énarchista, los primeros egresados como administradores públicos acometerían importantes tareas ejecutivas en los distintos niveles de la Administración Pública. Su preparación en el manejo de los

Para una visión comparativa ver el número especial "The Evolution of Political Science: Selected Case Studies" (1987) y Easton, Gunnell y Graciano (1991). 
asuntos de Estado, incluía nociones sobre sistemas políticos, partidos políticos y política internacional. Con énfasis en el funcionamiento formal y legal de las instituciones políticas, estas materias contribuyeron a formar buenos generalistas y especialistas en temas de la administración del Estado. Pero al poner énfasis en la práctica de los principios de la administración, la investigación y producción de conocimiento en ciencia política no tuvo prioridad².

Fue sólo con el advenimiento de la revolución conductista en la ciencia política norteamericana en los años 60 que la disciplina adquirió autonomía en Chile y una orientación hacia la investigación. Un rol destacado le cabe en este movimiento a la Facultad Latinoamericana de Ciencias Sociales (FLACSO), cuya sede en Santiago de Chile comenzó a formar como cientistas políticos, desde mediados de los años 60 , a profesionales de toda Latinoamérica a nivel de postgrado. La formación en la Escuela Latinoamericana de Ciencia Política y Administración Pública (ELACP) incluía una sólida preparación en teórica política, metodología de la investigación y estadística³ ${ }^{3}$ Asimismo, ELACP comenzó a publicar la Revista Latinoamericana de Ciencia Política en 1970, donde se incluyeron importantes trabajos de cientistas políticos latinoamericanos emergentes y norteamericanos ${ }^{4}$.

La Universidad de Chile crea el Instituto de Estudios Internacionales en 1966, dedicado a la investigación y a impartir un postgrado en Relaciones Internacionales, atrayendo un número importante de estudiantes latinoamericanos. El Instituto publica la revista Estudios Internacionales que se transforma, desde entonces, en el único referente especializado sobre el tema en Chile. La Pontificia Universidad Católica de Chile (PUC) funda un Departamento de Ciencia Política en 1969, el cual se transforma en 1970 en Instituto de ciencia política. Con la implementación de un Diplomado en Relaciones Internacionales en 1972, el Instituto se transforma en el primer centro universitario abocado al desarrollo de la ciencia política como una disciplina autónoma y en todas sus especialidades 5 . Sin embargo, no será sino hasta 1982, diez años después, cuando el Instituto pondrá en marcha su programa de Magíster en Ciencia Política y comenzará, en 1979, a publicar la Revista de Ciencia Política, la primera en su género en el país.

Estos centros académicos universitarios surgieron con el importante apoyo de convenios con organismos internacionales, fundaciones y universidades norteamericanas, que apoyaron la expansión del "credo conductista" hacia las ciencias sociales en América Latina y en el contexto de un importante proceso de movilización social, de agudización de los conflictos ideológicos y de una confrontación paradigmática en las ciencias sociales.

Para Marcos Kaplan, la ciencia política latinoamericana se movió en la segunda mitad de los 60 entre extremos paradigmáticos que promovieron por un lado un "conformismo riguroso, una mentalidad rígidamente disciplinada, desfavorable a la vida intelectual, a la crítica y a la investigación, que se contrapuso a toda hipótesis", resultando en la "degradación teórica, la limitación metodológica y técnica". Y, por otro lado, un positivismo que promovió la "neutralidad valorativa, identifi-

2 Agrupados en el Colegio Profesional de Administradores Públicos, debe reconocerse que la Revista Chilena de Administración Pública, publicada por dicha asociación, promovió la investigación teórica y aplicada en el ámbito de la administración pública. Para una relación entre la ciencia política y la administración pública, ver Rehren (1993: 17-19).

3 Ver el Programa Docente y Programa de Investigaciones en Revista Latinoamericana de Ciencia Política 2: 556-605.

$4 \quad$ La Revista Latinoamericana de Ciencia Política tuvo una publicación de tres volúmenes con seis números desde 1970 hasta 1973.

5 Para una breve reseña histórica del Instituto de Ciencia Política, ver Programa de Magíster en Ciencia Política (2002). 
cada con el rigor científico, desligada de valores, orientaciones y compromisos sociales y políticos" (Kaplan, 1970: 28-30 y 37-38). A pesar de esta tensión, los inicios de la ciencia política chilena en los años 60 fueron excepcionalmente auspiciosos desde una perspectiva institucional.

Durante el período autoritario (1973-1989) y ante un aparente congelamiento del desarrollo de la disciplina, la emergencia de centros académicos e intelectuales independientes y paralelos a las universidades, lograron dar un impulso y mantener a la ciencia política como una disciplina que podía, bajo su alero protector, generar conocimiento e investigación independiente del régimen. Centros como la Corporación de Investigaciones Económicas para Latinoamérica (CIEPLAN), el Centro para el Estudio de la Realidad Contemporánea (CERC) y la Corporación de Promoción Universitaria (CPU), implementaron importantes proyectos con apoyo de fundaciones norteamericanas y europeas y publicaron revistas como la Colección Estudios Cieplan, Opciones y Estudios Sociales respectivamente, dando cuenta en ellas de los resultados de sus investigaciones.

Sin embargo, producido el retorno a la democracia, tanto CIEPLAN como CERC dejaron de tener gravitación y sus publicaciones se descontinuaron. Sólo CPU se mantuvo activo y Estudios Sociales continuó publicándose periódicamente, con un importante cambio en su línea editorial favoreciendo el estudio de las políticas públicas. Asimismo, hacia fines de la década de los 80 , el Instituto Latinoamericano de Doctrina y Estudios Sociales (ILADES), hoy Universidad Alberto Hurtado, comenzó a publicar Persona y Sociedad, revista que concentra la mayor parte de sus artículos en temas de ética vinculados a la política, la economía y la sociedad, e implementó un Magíster en Estudios Sociales y Políticos Latinoamericanos en 1995.

Dos importantes nuevos centros de estudio emergerán a principios de los 80 . El Instituto de Ciencia Política de la Universidad de Chile, creado en 1982, iniciará un Programa de Magíster y publicará la revista Política, constituyéndose en el tercer centro universitario dedicado al fomento de la disciplina. El sector empresarial por su parte creará el influyente Centro de Estudios Públicos y editará la revista Estudios Públicos, donde se publicarán importantes artículos vinculados a todas las especialidades de la disciplina y a fomentar las ideas liberales, tanto en el ámbito filosófico, político como económico.

La institucionalización de la ciencia política culmina con la creación de la Asociación Chilena de Ciencia Política en 1986, que en la actualidad reúne entre 150 a 200 miembros asociados entre profesores y estudiantes ${ }^{6}$. Su principal actividad es la difusión del estudio de la disciplina y la organización de los Congresos Nacionales de Ciencia Política, de los cuales se han efectuado seis versiones hasta el año 2004.

Podemos afirmar que durante los años 80 y en los albores de la transición a la democracia, existe ya una infraestructura institucional capaz de generar un desarrollo sostenido de la ciencia política en Chile. Así lo demostrarán la reciente creación de carreras de pre y postgrado en ciencia política en varias universidades públicas y privadas del país y una creciente demanda de alumnos por seguir ya sea una licenciatura o cursar distintos ramos ofrecidos en las mallas curriculares de la disciplina. 


\section{CONSIDERACIONES METODOLÓGICAS}

Con el fin de responder a las preguntas planteadas en la introducción de este trabajo y de analizar los datos recogidos en las tres dimensiones consideradas como claves para el desarrollo de la disciplina - publicaciones, tesis e investigaciones- se consideraron las siguientes áreas de especialización y sub-especialización propias de la ciencia política:

1. Teoría Política: teoría democrática y ética y política.

2. Instituciones y Procesos Políticos: sistemas políticos, partidos políticos, transiciones, constituciones, fuerzas armadas, presidencia y parlamentos.

3. Comportamiento Político: sistemas electorales, elecciones y plebiscitos.

4. Políticas Públicas: políticas públicas generales y políticas sectoriales.

5. Relaciones Internacionales: sistema internacional, política exterior de Chile, relaciones interamericanas, relaciones internacionales en Europa, América Latina, URSS, África y Asia.

6. Historia Política: historia política de Chile, historia política de América Latina.

Las revistas analizadas fueron aquellas publicadas por centros y/o institutos académicos vinculados directamente a la disciplina y que mantuvieron continuidad en sus ediciones en el período 1980-2000 (ver Tabla 1). Cada artículo publicado fue clasificado en una de las categorías mencionadas anteriormente según el título del mismo y un análisis de su contenido que corroborara la afinidad con el título (ver Tabla 2).

TABLA 1: Revistas de ciencia política (o vinculadas a la disciplina) publicadas en Chile

\begin{tabular}{|c|c|c|c|}
\hline Institución & Revista & Desde & Hasta \\
\hline $\begin{array}{l}\text { Facultad Latinoamericana de } \\
\text { Ciencias Sociales (FLACSO) }\end{array}$ & $\begin{array}{l}\text { Revista Latinoamericana de } \\
\text { Ciencia Política (descontinuada) }\end{array}$ & 1970 & 1973 \\
\hline $\begin{array}{l}\text { Instituto de Estudios Internacionales, } \\
\text { Universidad de Chile }\end{array}$ & Estudios Internacionales & 1967 & Hoy \\
\hline $\begin{array}{l}\text { Instituto de Ciencia Política, Pontificia } \\
\text { Universidad Católica de Chile }\end{array}$ & Revista de Ciencia Política & 1979 & Hoy \\
\hline $\begin{array}{l}\text { Corporación de Investigaciones } \\
\text { Económicas para Latinoamérica } \\
\text { (CIEPLAN) }\end{array}$ & $\begin{array}{l}\text { Colección Estudios Cieplan } \\
\text { (descontinuada) }\end{array}$ & 1979 & 1997 \\
\hline $\begin{array}{l}\text { Centro para el Estudio de la Realidad } \\
\text { Contemporánea (CERC) }\end{array}$ & $\begin{array}{l}\text { Opciones } \\
\text { (descontinuada) }\end{array}$ & 1983 & 1989 \\
\hline $\begin{array}{l}\text { Corporación de Promoción } \\
\text { Universitaria (CPU) }\end{array}$ & Estudios Sociales & 1973 & Hoy \\
\hline $\begin{array}{l}\text { Instituto Latinoamericano de Doctrina } \\
\text { y Estudios Sociales (ILADES) }\end{array}$ & Persona y Sociedad & 1987 & Hoy \\
\hline $\begin{array}{l}\text { Instituto de Ciencia Política, } \\
\text { Universidad de Chile }\end{array}$ & Política & 1982 & Hoy \\
\hline Centro de Estudios Públicos & Estudios Públicos & 1980 & Hoy \\
\hline
\end{tabular}


TABLA 2: Publicaciones de ciencia política en Chile: 1980-2000 (por revista y especialidad)

\begin{tabular}{|c|c|c|c|c|c|c|c|}
\hline $\begin{array}{c}\text { ÁREA DE } \\
\text { ESPECIALIDAD }\end{array}$ & $\begin{array}{l}\text { Revista } \\
\text { Ciencia } \\
\text { Política }\end{array}$ & Política & $\begin{array}{l}\text { Estudios } \\
\text { Interna- } \\
\text { cionales }\end{array}$ & $\begin{array}{l}\text { Estudios } \\
\text { Públicos }\end{array}$ & $\begin{array}{l}\text { Estudios } \\
\text { Sociales }\end{array}$ & $\%$ & (N) \\
\hline Teoría Política & 17 & 34 & & 90 & 29 & 12,4 & (170) \\
\hline Teoría Democrática & 13 & 10 & 1 & 27 & 24 & 5,5 & (75) \\
\hline Ética y Política & 7 & 1 & & 1 & 2 & 0,8 & (11) \\
\hline TEORÍA POLITICA & 37 & 45 & 1 & 118 & 55 & 18,7 & $(256)$ \\
\hline Sistemas Políticos & 5 & 2 & & 4 & 13 & 1,7 & (24) \\
\hline Partidos Políticos & 12 & 11 & 1 & 21 & 10 & 4,0 & (55) \\
\hline Transiciones & 20 & 2 & 4 & 16 & 17 & 4,3 & (59) \\
\hline Constitución & 5 & 6 & & 10 & 4 & 1,8 & (25) \\
\hline Fuerzas Armadas & 9 & 3 & & 1 & 2 & 1,1 & (15) \\
\hline Presidencia & 8 & 0 & & 0 & 6 & 1,0 & (14) \\
\hline Parlamento & 7 & 0 & & 0 & 1 & 0,6 & (8) \\
\hline INST. Y PROCESOS & 66 & 24 & 5 & 52 & 53 & 14,5 & $(200)$ \\
\hline Sistemas Electorales & 10 & 9 & 0 & 3 & 3 & 1,8 & (25) \\
\hline Elecciones & 0 & 5 & 0 & 23 & 1 & 2,1 & (29) \\
\hline COMP. POLITICO & 10 & 14 & & 26 & 4 & 3,9 & (54) \\
\hline POLITICAS PÚBLICAS & 19 & 9 & 0 & 45 & 73 & 10,6 & (146) \\
\hline Sistema Internacional & 14 & 14 & 51 & 3 & 19 & 7,4 & $(101)$ \\
\hline Política Exterior Chile & 23 & 7 & 33 & 3 & 2 & 5,0 & (68) \\
\hline RR.II. EEUU-Am. Lat. & 0 & 6 & 25 & 2 & 1 & 2,5 & (34) \\
\hline RR.II. A. Latina & 5 & 25 & 192 & 13 & 27 & 19,0 & (262) \\
\hline RR.II. Europa & 0 & 6 & 34 & 9 & 11 & 4,4 & $(60)$ \\
\hline RR.II. URSS & 2 & 8 & 7 & 16 & 1 & 2,5 & (34) \\
\hline RR.Il. África & 1 & 2 & 1 & 1 & 0 & 0,4 & (5) \\
\hline RR.II. Asia & 1 & 0 & 12 & 1 & 0 & 1,0 & (14) \\
\hline RR.II. EE.UU & 6 & 0 & 10 & 1 & 0 & 1,2 & (17) \\
\hline REL. INTERNACIONALES & 552 & 68 & 365 & 49 & 61 & 43,4 & (595) \\
\hline Historia Política Chile & 6 & 15 & 0 & 60 & 27 & 7,9 & $(108)$ \\
\hline Hist. Política A. Latina & 1 & 15 & 0 & 3 & 7 & 1,9 & (26) \\
\hline HISTORIA POLITICA & 7 & 30 & 0 & 63 & 34 & 9,8 & (134) \\
\hline TOTAL & 191 & 190 & 354 & 352 & 286 & 100,0 & (1373) \\
\hline
\end{tabular}




\section{LAS PUBLICACIONES EN LAS REVISTAS DE CIENCIA POLÍTICA CHILENA}

Los datos de la Tabla 2 reflejan que durante el período 1980-2000 se han publicado un total de 1.373 artículos. De las áreas de especialidad, la más importante es la de relaciones internacionales, que concentra un $43,3 \%$ del total de artículos publicados. Las otras especialidades están todas situadas muy por debajo del nivel de productividad de esta área. Llama la atención la escasa visibilidad de áreas como comportamiento electoral y políticas públicas que en países donde la disciplina se encuentra más desarrollada, concentran gran cantidad de publicaciones. La escasa formación en metodología y técnicas cuantitativas es enciales para la investigación en estas áreas, como también el hecho de que las políticas públicas empiezan a adquirir importancia solamente durante la década de los 90, explican esta situación. Por ejemplo, el 75\% del total de los artículos de políticas públicas se publican durante esta década.

Las relaciones internacionales de América Latina concentran el $19 \%$ del total de publicaciones del período $1980-2000$ y cerca del $50 \%$ de todas las publicaciones del área de relaciones internacionales, superando incluso aquellas áreas de especialidad tradicionales como teoría política, instituciones y procesos, políticas públicas y comportamiento político. Del mismo modo, teoría política, con temas de teoría política clásica, moderna y/ o contemporánea, concentra alrededor del $60 \%$ de las publicaciones al interior del área. En otras áreas de especialidad tradicionales, como instituciones y procesos, no existe un tema predominante. Sin embargo, los artículos sobre historia política de Chile concentran el $8 \%$ del total de publicaciones, lo cual no es irrelevante, si lo comparamos por ejemplo, con el área de comportamiento político. Lo anterior refleja probablemente la estrecha vinculación de la ciencia política chilena con otras disciplinas de las ciencias sociales, con las cuales estuvo originalmente ligada.

Quizás lo más destacable que muestra la Figura 2 es la especialización de las revistas chilenas, hecho que también se puede apreciar en la Figura 1. El caso más notorio es el de Estudios Internacionales que concentra el $61 \%$ de todas las publicaciones del área de relaciones internacionales del país. El 33\% de las publicaciones en instituciones y procesos han aparecido en la Revista de Ciencia Política y el $50 \%$ de las publicaciones de políticas públicas aparecen en Estudios Sociales. Por otro lado, Estudios Públicos concentra el $46 \%$ de las publicaciones del área de teoría política, el $48 \%$ en comportamiento político y el $47 \%$ de todos los artículos publicados en historia política, demostrando así la gravitación de esta revista en la disciplina. 
FIGURA 1: Total de articulos publicados por revista y especialidad (1980-2000)

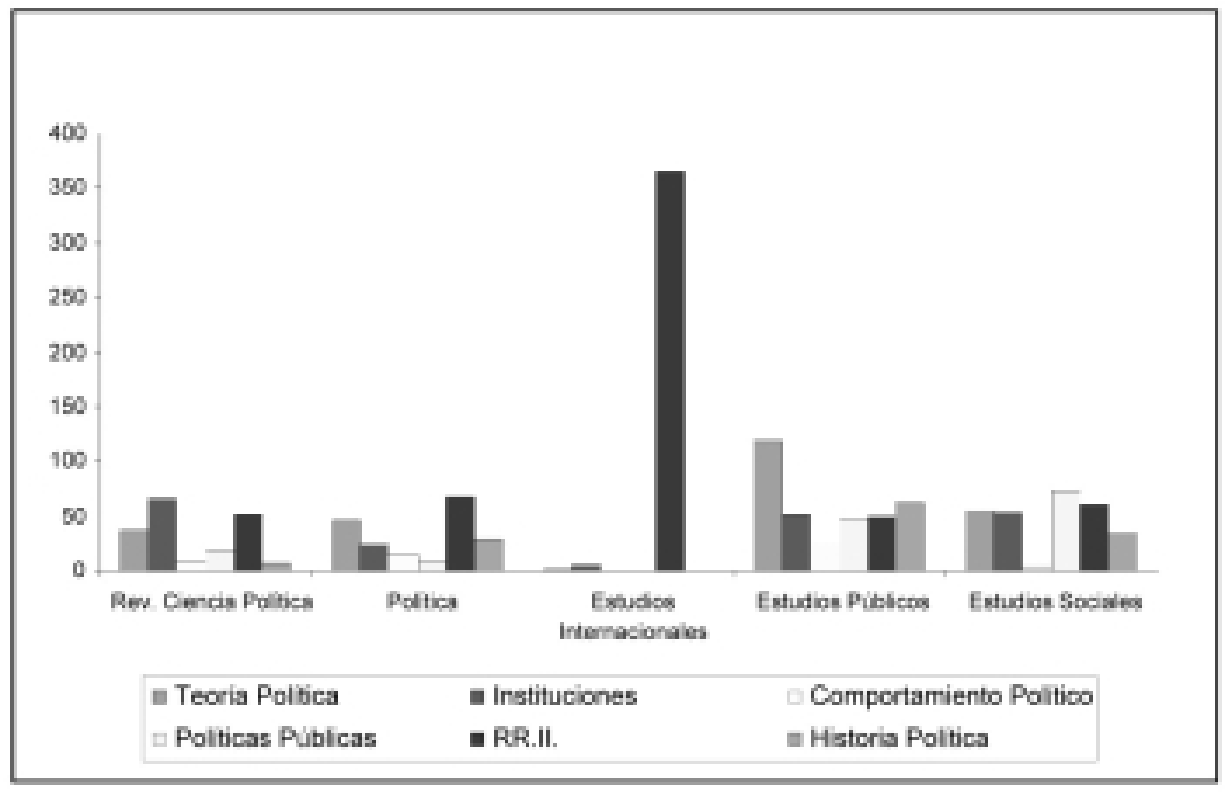

La Figura 2 también muestra que las publicaciones en la disciplina han sido fluctuantes. A esto contribuye el aumento explosivo del número de artículos publicados en ciertos años por algunas revistas. Por ejemplo, durante 1982-83 y 1987-88 Política aumentó notoriamente el número de artículos publicados. Asimismo, durante 1993-94, el incremento correspondió a Estudios Públicos y Estudios Internacionales, tendencias que pueden ser fácilmente observables en la Figura 3. Además, se observa una tendencia a la disminución del número total de artículos publicados durante la década de los noventa en comparación con los 80 . En promedio, la única revista que aumentó su número de artículos en los 90, fue Estudios Públicos, que subió en promedio de 13 a 20 artículos por año. 
FIGURA 2: Total de Artículos Publicados 1980-2000

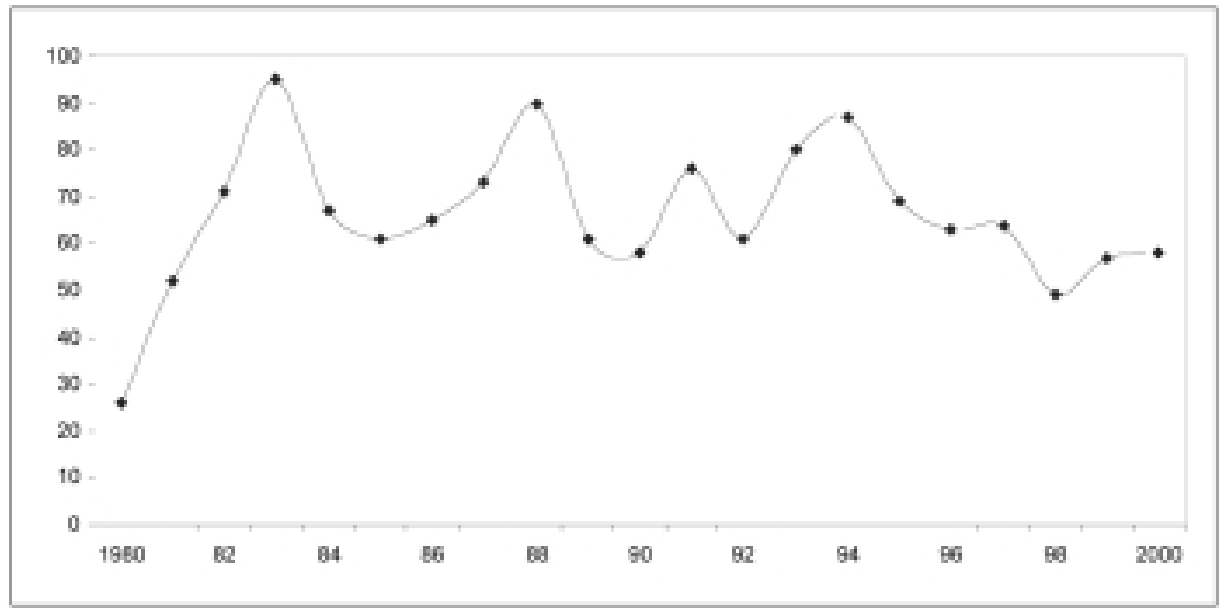

FIGURA 3: Evolución del numero de artículos publicados por revista 1980-2000

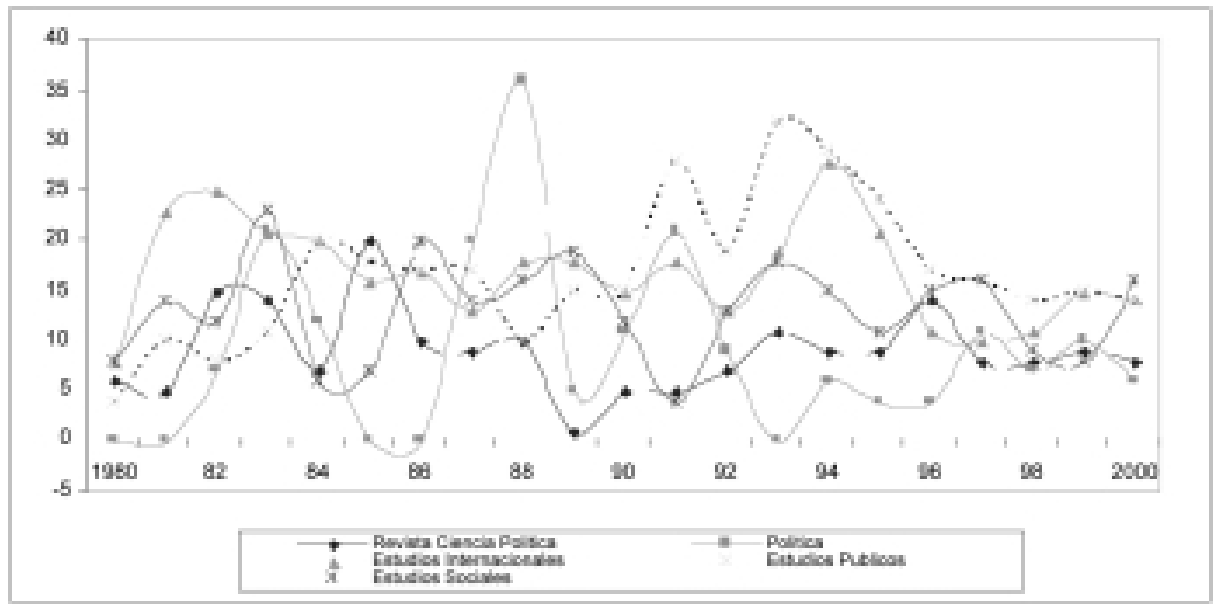

Finalmente, es posible establecer un perfil histórico de las revistas en base a sus publicaciones 1980-2000. Así, la Revista de Ciencia Política concentra un 37\% de todas sus publicaciones en instituciones y procesos políticos, seguido de relaciones internacionales con un $27 \%$ y teoría política con un $19 \%$. Política concentra un $36 \%$ de sus publicaciones en relaciones internacionales, seguido de teoría política con un $24 \%$, historia política con un $16 \%$ e instituciones y procesos con un 13\%. El 98\% de los artículos de Estudios Internacionales están en relaciones internacionales. En Estudios Públicos el 33\% de todos sus artículos corresponde a teoría política, seguido de historia política con un $18 \%$ y políticas públicas con un $12 \%$. Finalmente, Estudios Sociales publica un $26 \%$ de sus artículos en políticas públicas, seguido por relaciones internacionales y teoría política con un $21 \%$ e instituciones y procesos con un $19 \%$. 
Una comparación longitudinal nos muestra que las revistas también experimentan cambios en la naturaleza de los artículos que publican. Así por ejemplo, en la Revista de Ciencia Política un 74\% de los artículos en políticas públicas aparecieron en la década de los 90. Por otro lado, Política publicó el $72 \%$ de sus artículos en comportamiento electoral en los 90 . En Estudios Públicos el $72 \%$ de sus artículos en historia política y políticas públicas aparecen en la misma década. Finalmente, Estudios Sociales publica el $73 \%$ de sus artículos en políticas públicas en ese periodo.

\section{LA PRODUCTIVIDAD DE POSTGRADO: TESIS DE MAGÍSTER EN CIENCIA POLÍTICA}

Las tesis de magíster constituyen en la disciplina el instrumento formal de graduación de los cientistas políticos. Asimismo, reflejan los intereses de investigación de las nuevas generaciones y el expertise de los profesores ya establecidos en algunas áreas de la disciplina. A este respecto, es posible hipotetizar que existe alguna relación entre lo publicado por los investigadores, sus experiencias y las tesis de los alumnos que dirigen. Además, los temas de tesis normalmente están influidos por las prioridades establecidas en la agenda pública. Desde esta perspectiva, la tesis es una concreción empírica de una realidad política nacional, del estado de la disciplina, sus avances, vacíos y debilidades.

La Tabla 3 nos muestra que de las 226 tesis aprobadas entre 1980-2000, el 38\% se concentró en relaciones internacionales, siendo el área más activa en la disciplina, seguida por instituciones y procesos políticos con un $24 \%$ y políticas públicas con un $16 \%$ del total de tesis elaboradas. Llama la atención el bajo número de tesis realizadas en comportamiento electoral, situación que coincide con las escasas publicaciones de la misma área, tal como se analizó en la sección anterior.

TABLA 3: Total tesis magíster en ciencia política: 1980-2000 (por área de especialidad)

\begin{tabular}{lcc}
\hline ÁREAS DISCIPLINARIAS & Porcentaje & N \\
\hline Teoría Política & 13,7 & 31 \\
Instituciones y Procesos Políticos & 24,3 & 55 \\
Comportamiento Electoral & 1,3 & 3 \\
Políticas Públicas & 15,5 & 35 \\
Relaciones Internacionales & 37,6 & 85 \\
Historia Política & 7,5 & 17 \\
TOTAL TESIS & 100,0 & 226 \\
\hline
\end{tabular}

Una comparación institucional de las tesis (ver Tabla 4) nos muestra que en la Pontificia Universidad Católica de Chile, el $51 \%$ de ellas se concentra en relaciones internacionales y el $26 \%$ en instituciones y procesos, mientras que la Universidad de Chile tiene una distribución más equilibrada entre relaciones internacionales, instituciones y procesos, teoría política y políticas públicas. Asimismo, el número de tesis elaboradas en teoría política en la Pontificia Universidad Católica de Chile es bajo, mientras que existe un número más alto de tesis en historia política en la Universidad de Chile ${ }^{7}$.

No se contó con los datos de las tesis de magíster del Instituto de Estudios Internacionales de la Universidad de Chile. Sin embargo, podemos asumir, dado el patrón de publicaciones de la revista y especialización de dicho Instituto, que la mayoría de ellas fueron realizadas en el área de relaciones internacionales. 
TABLA 4: Tesis de magíster en ciencia política: 1980-2000 (por instituto y área de especialidad)

\begin{tabular}{lrrrr}
\hline & \multicolumn{2}{c}{ PUC } & \multicolumn{2}{c}{ U. de Chile } \\
\hline ÁREAS DISCIPLINARIAS & \multicolumn{1}{c}{$(\%)$} & $\mathrm{N}$ & \multicolumn{1}{c}{$(\%)$} & $\mathrm{N}$ \\
\hline Teoría Política & 7,3 & $(8)$ & 19,7 & $(23)$ \\
Instituciones y Procesos Políticos & 25,7 & $(28)$ & 23,1 & $(27)$ \\
Comportamiento Electoral & 1,8 & $(2)$ & 0,9 & $(1)$ \\
Políticas Públicas & 11,9 & $(13)$ & 18,8 & $(22)$ \\
Relaciones Internacionales & 51,4 & $(56)$ & 24,8 & $(29)$ \\
Historia Política & 1,8 & $(2)$ & 12,8 & $(15)$ \\
TOTAL TESIS & 100 & $(109)$ & 100 & $(117)$ \\
\hline
\end{tabular}

Una perspectiva longitudinal muestra que la mayor proporción de las tesis se realiza en los períodos comprendidos entre 1989-1991 en una primera fase y a continuación entre 1996-98 en una segunda fase. En el primer periodo el aumento corresponde significativamente al área de relaciones internacionales. En el segundo, el aumento en las tesis es notorio no sólo en relaciones internacionales, sino que también en el área de instituciones y procesos políticos y políticas públicas, áreas que representan un crecimiento sustantivo (ver Figura 4). Parte importante de esta tendencia se debe al aumento del número de tesis aprobadas en la Pontificia Universidad Católica de Chile por sobre el promedio histórico, especialmente entre los años 1996-98, tal como lo muestra la Figura 5 . En dicho período se realizaron el $40 \%$ del total de las tesis de magíster del Instituto de Ciencia Política de la PUC.

FIGURA 4: Evolución tesis de magíster por área de especialidad (1980-2000)

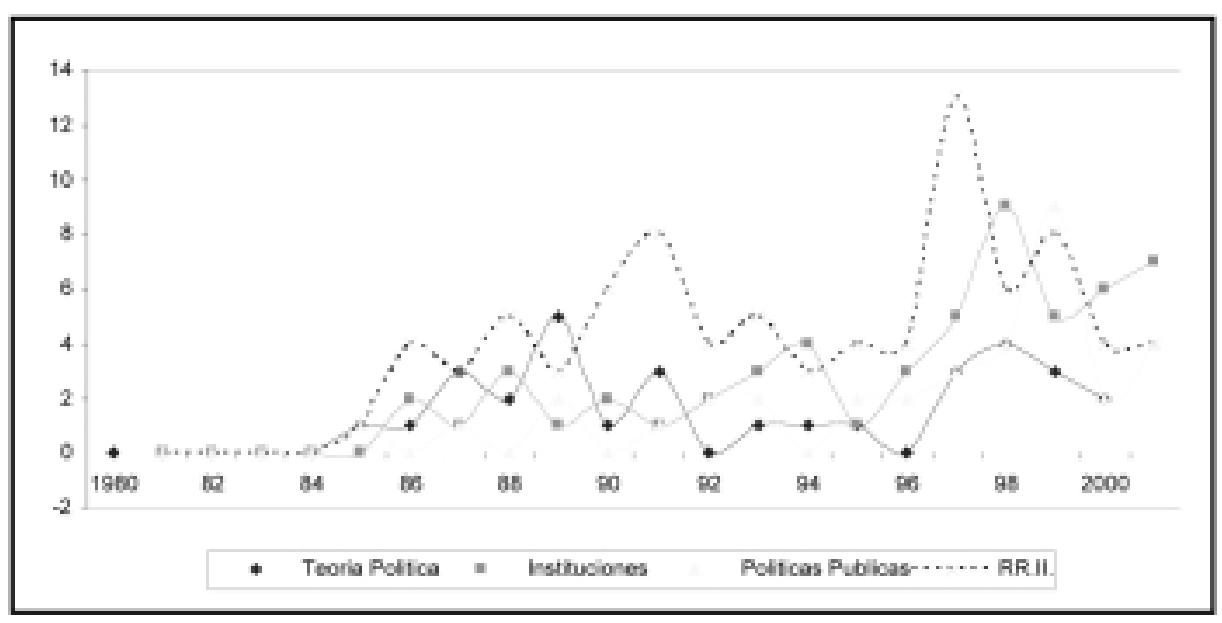


FIGURA 5: Número de tesis de magíster por instituto 1980-2000

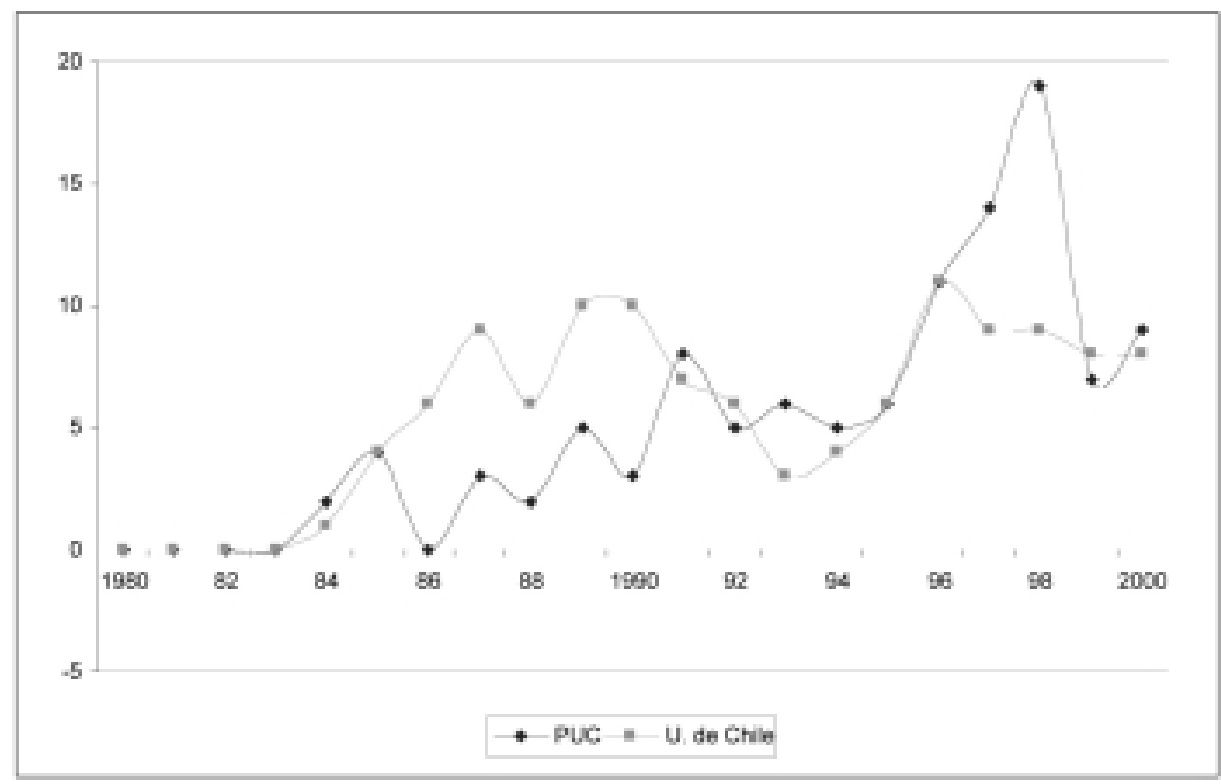

\section{LA INVESTIGACIÓN EN CIENCIA POLÍTICA}

Una disciplina nueva como la ciencia política no gozó de flujos regulares de recursos que apoyaran su desarrollo. En los años 60 fueron convenios internacionales con universidades y fundaciones extranjeras los que financiaron, a través de proyectos de investigación concretos, el incipiente desarrollo de la disciplina. Con posterioridad, la creación de direcciones de investigación al interior de las universidades permitió canalizar recursos escasos hacia ella. Sólo con la creación del Fondo de Desarrollo Científico y Tecnológico (FONDECYT) en 1982, se institucionalizó el financiamiento de la disciplina por parte del Estado. La apertura de concursos para el financiamiento de proyectos abrió una posibilidad cierta y regular que contribuía a la estabilidad y continuidad de la investigación. Por lo tanto, resulta vital establecer hasta qué punto la Ciencia Políica ha aprovechado las oportunidades de financiamiento y pasa de una etapa de desarrollo incipiente a una fase de desarrollo autosostenido.

Una primera evaluación efectuada en 1992, estableció que "la participación de la disciplina de la Ciencia Políica [en FONDECYT] ha sido particularmente poco significativa" (Urrutia, 1992: 9). La Figura 6 muestra que en términos generales dicha situación se mantuvo hasta 1990 con escasos proyectos aprobados y ninguno aprobado para el mismo año. Sin embargo, esta situación se revierte a contar de 1991-92, cuando el número de proyectos aprobados llega a su máximo histórico con un total de 10 proyectos aprobados, cinco de los cuales eran de política exterior chilena. Dicho tema de investigación representa el $30 \%$ de todos los proyectos aprobados durante 1982-2000, tal como lo muestra la Tabla 5. Por otra parte, la aprobación de proyectos en instituciones y procesos políticos es significativa a contar de 1990. De hecho hasta 1990 sólo se habían aprobado proyectos en el área de relaciones internacionales y ninguno en instituciones y proce- 
sos. Los 13 proyectos que aparecen aprobados en instituciones y procesos lo fueron entre 19912000 , con siete de ellos vinculados al tema de sistemas políticos. Cabe destacar la baja aprobación de proyectos en las áreas de teoría política y comportamiento electoral.

A pesar de la mayor participación de la ciencia política en FONDECYT a contar de los 90, el porcentaje de proyectos aprobados fue de tan solo un $1 \%$ del total de 6.542 aprobados durante 1982-2000 por dicho fondo, situación que contrasta con el 11,1\% aprobado para Ciencias Sociales y J urídicas ${ }^{8}$.

FIGURA 6: Número de proyectos FONDECYT aprobados en ciencia política, 1980-2000

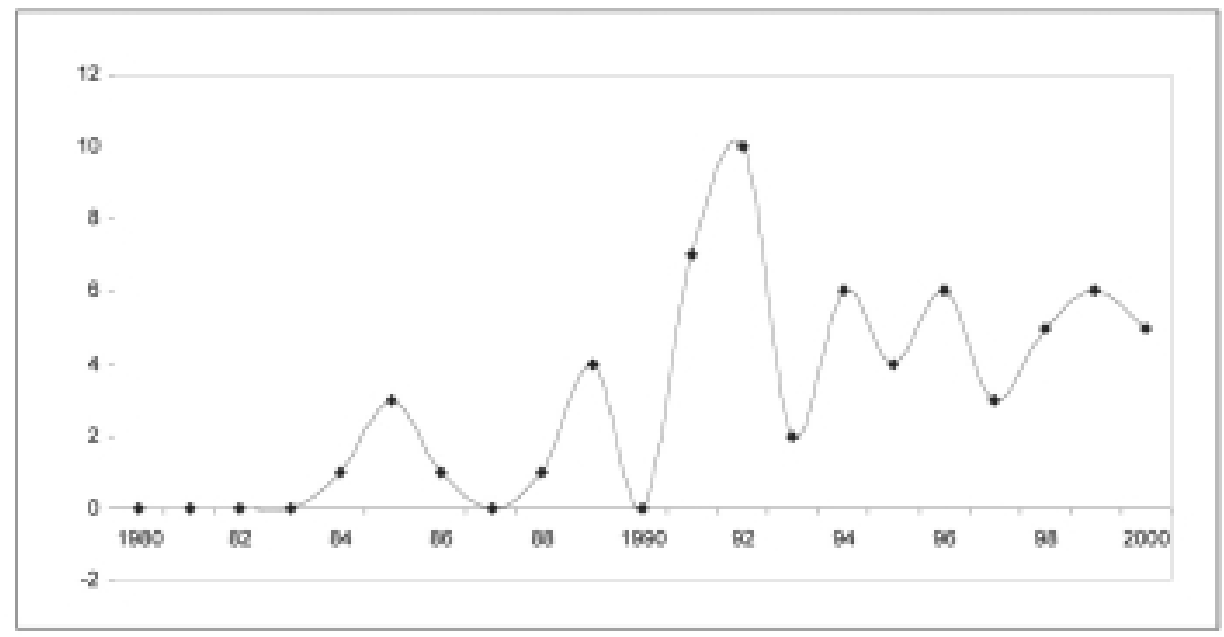

\section{CONCLUSIONES}

El análisis precedente permite concluir que la Ciencia Política en Chile está en un proceso de consolidación institucional, con programas de postgrado regulares que producen un flujo constante de tesis de Magíster, financiamiento estable y revistas ya establecidas, pero que necesitan mayor regularidad en sus publicaciones.

Las revistas publicadas por institutos universitarios muestran un alto grado de permanencia en el tiempo, reflejando el compromiso y requisito institucional universitario de divulgación, como parte de sus funciones académicas. La regularidad de Estudios Públicos y Estudios Sociales, en el ámbito de centros privados no universitarios como el Centro de Estudios Públicos y la Corporación de Promoción Universitaria respectivamente, reflejan el fuerte compromiso de estos centros con el estudio de la realidad socio-económica y política nacional, contribuyendo en forma adicional al desarrollo de la disciplina en Chile.

Una revisión del desarrollo por áreas de especialización, medida en publicaciones, número de tesis y proyectos de investigación durante el período 1980-2000, muestra un desarrollo desigual. Áreas como comportamiento político y políticas públicas, a pesar del desarrollo reciente de 
esta última en los años 90, están relativamente atrasadas con respecto a otras áreas de la disciplina y no siguen las tendencias de la ciencia política actual a nivel internacional.

Asimismo, existe una asimetría intra-área al observar la relación entre los componentes mencionados en el párrafo anterior. Por ejemplo, el grado de participación de relaciones internacionales en el porcentaje del total de publicaciones es inferior a su participación en el total de proyectos FONDECYT aprobados y lo mismo puede decirse para instituciones y procesos y políticas públicas, aunque en menor grado. Lo anterior debe mover a un análisis de la relación número de proyectos de investigación/ publicaciones, al menos en el ámbito nacional. Una tendencia opuesta se observa en teoría política, historia política y comportamiento político, donde existe una mayor participación en el total de publicaciones y tesis de magíster que en la participación de estas áreas en el total de proyectos FONDECYT aprobados.

Un ranking por áreas de la ciencia política chilena, construido en base a los datos analizados en este trabajo, entrega los siguientes resultados para el período 1980-2000:

TABLA 5: $\quad$ Ranking por áreas de la ciencia política chilena

\begin{tabular}{lccc}
\hline \multicolumn{1}{c}{ ÁREAS DE ESPECIALIZACIÓN } & $\begin{array}{c}\text { \% proyectos } \\
\text { FONDECYT } \\
\text { aprobados }\end{array}$ & $\begin{array}{c}\text { \% de Tesis de } \\
\text { Magíster }\end{array}$ & $\begin{array}{c}\text { \% total de } \\
\text { publicaciones }\end{array}$ \\
\hline Relaciones Internacionales & 62,5 & 37,6 & 43,4 \\
Instituciones y Procesos Políticos & 20,5 & 24,3 & 14,5 \\
Políticas Públicas & 12,5 & 15,5 & 10,6 \\
Teoría Política & 3,0 & 13,7 & 9,8 \\
Historia Política & 0,0 & 7,5 & 9,8 \\
Comportamiento Político & 1,6 & 1,3 & 3,9 \\
\hline
\end{tabular}


TABLA 6: Proyectos FONDECYT aprobados por área (1980-2000)

\begin{tabular}{|c|c|c|}
\hline ÁREAS DISCIPLINARIAS & Total & Porcentaje \\
\hline Teoría Política & 0 & 0,0 \\
\hline Teoría Democrática & 1 & 1,6 \\
\hline Ética y Política & 1 & 1,6 \\
\hline TEORÍA POLITICA & 2 & 3,2 \\
\hline Sistema Político & 7 & 10,9 \\
\hline Partidos Políticos & 1 & 1,6 \\
\hline Transición a la democracia & 1 & 1,6 \\
\hline Constitución & 0 & 0,0 \\
\hline Fuerzas Armadas & 0 & 0,0 \\
\hline Presidencia & 2 & 3,2 \\
\hline Parlamento & 2 & 3,2 \\
\hline INSTITUCIONES Y PROCESOS & 13 & 20,5 \\
\hline Sistema Electoral & 0 & 0,0 \\
\hline Elecciones y Plesbicito & 1 & 1,6 \\
\hline COMPORTAMIENTO ELECTORAL & 1 & 1,6 \\
\hline POLITICAS PÚBLICAS & 8 & 12,5 \\
\hline Sistema Internacional & 6 & 9,3 \\
\hline Política Exterior de Chile & 19 & 29,7 \\
\hline Relaciones Estados Unidos- A. Latina & 3 & 4,7 \\
\hline RR. II. en América Latina & 11 & 17,2 \\
\hline RR. II. en Europa & 1 & 1,6 \\
\hline RR. II. en URSS & 0 & 0,0 \\
\hline RR. II. en África & 0 & 0,0 \\
\hline RR. II. en Asia & 0 & 0,0 \\
\hline RELACIONES INTERNACIONALES & 40 & 62,5 \\
\hline Historia Política de Chile & 0 & 0,0 \\
\hline Historia Política de América Latina & 0 & 0,0 \\
\hline HISTORIA POLITICA & 0 & 0,0 \\
\hline TOTAL C. POLÍTICA & 24 & 37,5 \\
\hline TOTAL RR. II. & 40 & 62,5 \\
\hline TOTAL PROYECTOS & 64 & 100,0 \\
\hline
\end{tabular}

Fuente: FONDECYT. 


\section{REFERENCIAS}

Easton, David, Gunnell John G. y Luigi Graciano (eds.). 1991. The Development of Political Science: A Comparative Survey. London: Routledge.

Fernández, María de los Ángeles. 2003. "Sombras nada más? Estado, mercado y ciencia política en el Chile contemporáneo". Anuario de Ciencias Politicas 1: 37-68.

Instituto de Ciencia Política, PUC. 2002. Programa Magíster de Ciencia Política. Santiago: Pontificia Universidad Católica de Chile.

Kaplan, Marcos. 1970. La ciencia política latinoamericana en la encrucijada. Santiago: Editorial Universitaria.

Rehren, Alfredo. 1993. "Ciencia política, administración pública y democracia". Boletín de la Asociación Chilena de Ciencia Política 1 (3): 17-19.

Urrutia, Ricardo. 1992. "La ciencia política en el Fondecyt". Boletín de la Asociación Chilena de Ciencia Política 1 (1): 7-10. Varios Autores. 1987. "The Evolution of Political Science: Selected Case Studies". International Political Science, Review 8 ( anuary).

Alfredo Rehren es Ph. D. Ciencia Política, University of Texas at Austin; y B.A. Ciencia Política, Allegheny College. Fue Coordinador de los Programas de Magíster en Ciencia Política en la Universidad de Chile y la Pontificia Universidad Católica de Chile, donde también se desempeñó como Sub-Director y Director del Instituto de Ciencia Política. Miembro del Grupo Asesor FONDECYT en Ciencia Política (1993-99) y ex-Secretario Ejecutivo de la Asociación Chilena de Ciencia Política.

(E-mail: arehren@puc.cl)

Marco Fernández es Licenciado en Historia, Universidad de Chile; Magíster en Ciencia Política, Pontificia Universidad Católica de Chile; y candidato a Doctor en Economía y Relaciones Internacionales, Universidad A. Madrid. Actualmente es Director de la Escuela de Ciencia Política de la Universidad Arcis.

(E-mail: mfernandez@universidadarcis.cl) 